Revista Interdisciplinaria de Humanidades, Educación, Ciencia y Tecnología

Año VII. Vol. VII. N²2. Edición Especial II. 2021

Hecho el depósito de ley: pp201602FA4721

ISSN-L: 2542-3029; ISSN: 2610-802X

Universidad Nacional Experimental Francisco de Miranda (UNEFM). Santa Ana de Coro. Venezuela

Ángela Aracely Andrade-Andrade; Tamara Vidal-Intriago; Grey Marisol Benavidez-Zambrano;

Esther Magdalena Gutiérrez-Pibaque

DOI 10.35381/cm.v6i2.546

\title{
Síndrome de Burnout en la profesión de enfermería en las instituciones de salud del Ecuador
}

\section{Burnout syndrome in the nursing profession in health institutions in Ecuador}

Ángela Aracely Andrade-Andrade

arac.1112@1hotmail.com

Universidad Estatal del Sur de Manabí, Jipijapa

Ecuador

https://orcid.org/0000-0002-1980-6894

Yeraldi Tamara Vidal-Intriago

yeanzarabe2010@hotmail.com

Universidad Estatal del Sur de Manabí, Jipijapa

Ecuador

https://orcid.org/0000-0003-1872-1298

Grey Marisol Benavidez-Zambrano

greysol2003@yahoo.com

Universidad Estatal del Sur de Manabí, Jipijapa

Ecuador

https://orcid.org/0000-0001-7214-065X

Esther Magdalena Gutiérrez-Pibaque estherguti83@hotmail.com

Universidad Estatal del Sur de Manabí, Jipijapa

Ecuador

https://orcid.org/0000-0001-9632-4832

Recibido: 10 de julio de 2021

Aprobado: 01 de octubre de 2021 
Ángela Aracely Andrade-Andrade; Tamara Vidal-Intriago; Grey Marisol Benavidez-Zambrano;

Esther Magdalena Gutiérrez-Pibaque

\title{
RESUMEN
}

El objetivo general de la investigación fue analizar el Síndrome de Burnout presente en la profesión de enfermería en las instituciones de salud del Ecuador, un importante talento humano al servicio de la colectividad y constante estrés. Desde el paradigma cuantitativo, la misma está enmarcada en un diseño documental-bibliográfico, ya que se analiza y estudia una serie de datos obtenidos relacionados al tema de investigación, alcanzados y registrados por otros investigadores. La población está constituida por tesis, revistas, trabajos arbitrados; fundamentando el contenido teórico. En relación a la técnica de análisis de contenido, permitió, generar la relación de las mismas, lo que permitió comparar y contrastar con la realidad y cumplir con el objetivo de la investigación. Se concluye que es necesario una reorientación de las actividades laborales para lograr un equilibrio emocional, evitar el estrés, y la sobre carga de trabajo evitando la aparición de los síntomas del Burnout.

Descriptores: Enfermedad profesional; trabajo; motivación; fatiga; salud. (Tesauro UNESCO)

\begin{abstract}
The general objective of the research was to analyze the Burnout Syndrome present in the nursing profession in the health institutions of Ecuador, an important human talent at the service of the community and constant stress. From the quantitative paradigm, it is framed in a documentary-bibliographic design, since a series of data obtained related to the research topic is analyzed and studied, reached and recorded by other researchers. The population is made up of theses, magazines, refereed works; supporting the theoretical content. In relation to the content analysis technique, it allowed to generate the relationship of the same, which allowed to compare and contrast with reality and fulfill the objective of the research. It is concluded that a reorientation of work activities is necessary to achieve emotional balance, avoid stress, and overload of work avoiding the appearance of burnout symptoms.
\end{abstract}

Descriptors: Professional illness; job; motivation; fatigue; health. (UNESCO Thesaurus). 


\section{INTRODUCCIÓN}

Los acelerados cambios y avances que ha experimentado la sociedad durante las últimas décadas cobran un significado para la profesión de enfermería un complejo escenario. La incorporación tecnológica, la formación y profesionalización, las reformas legales, las demandas familiares, la sobre carga laboral, la pandemia, y un sin número de nuevas variables provocan en los profesionales de la salud grados de inseguridad $y$ desconocimiento. Al respecto, Arias Gallegos y Muñoz del Carpio Toia (2016) explican:

En el grupo profesional de las enfermeras, estudios en Latinoamérica indican que la sobrecarga laboral, la organización del trabajo por turnos, el área de trabajo y las demandas familiares, tienen efectos negativos en el síndrome de burnout. En ese sentido, el género tiene impacto en la vulnerabilidad biológica, en la exposición y prevención de riesgos, así como en ciertas condiciones laborales como el acceso a recursos, la promoción, etc. (p.561)

Estos cambios componen la aparición de nuevos problemas de salud laboral como el estrés crónico, escenario que afecta directamente a un componente clave del proceso de salud: la enfermera(o). El profesional de enfermería debe estar en capacidad de brindar cuidados a todas las personas, independientemente de su condición; esta atención debe garantizar el bienestar y la seguridad de los individuos, preservando su salud. (De Arco Canoles y Suarez Calle, 2018, p.172).

En este sentido, uno de los problemas de salud laboral y que cobra relevancia en este siglo XXI, ha sido el llamado el Síndrome de Burnout o de desgate profesional, según Bosqued (2008,p.35), se exterioriza con cambios psicológicos que van acrecentando su intensidad desde el descontento y la irritación hasta estallidos emocionales, especialmente en profesionales con relación de sometimiento en el área de prestación de servicios, como es el caso de la enfermería y más aún si ejecutan sus labores inapropiadas, con turnos excesivos, inseguridad en el cargo, remuneración insuficiente y carencia de recursos materiales o humanos necesarios para una correcta ejecución de sus tareas.

Por consiguiente, el trabajo es un componente transcendental en el desarrollo del ser humano, ya que a través del mismo éste alcanza compensar sus requerimientos básicos, 
tales como: alimentación, vivienda, vestido, sustento, entre otros. Al mismo tiempo, permite al hombre orientar en la práctica sus conocimientos y actitudes, llevándolo a relacionarse y sentirse útil como individuo. De acuerdo, con Rodríguez (2005, p.12), el trabajo es la fuente esencial de la calidad de vida del hombre, argumentando que no hay calidad de vida sin trabajo, puesto que las necesidades materiales y gran parte de las espirituales sólo pueden satisfacerse con el trabajo, en suma, el trabajo es la manera ideal en la que el individuo puede obtener los medios económicos para cubrir sus necesidades y a su vez, favorecer el desarrollo personal.

Dicho proceso transformacional ha forjado en la masa trabajadora un mayor requerimiento hacia el desempeño de sus tareas, pero también ha ocasionado, en forma equivalente, frustraciones, insatisfacciones y la presencia del estrés negativo o distrés, derivando éstas exposiciones en un deficiente desempeño laboral. Estos factores, relacionados al entorno laboral, pudieran estar presentes en cualquier organización, pública o privada, independientemente al sector que pertenezca. Por esto, el cuidado de los otros, especialmente en la profesión de enfermería se ha relacionado con la presencia de estrés.

Al respecto, Vidotti, et al. (2019) manifiesta lo siguiente:

El ambiente de trabajo en las instituciones hospitalarias expone a los profesionales de enfermería, diariamente, a riesgos ocupacionales, sobre todo a los psicosociales, ya que están sometidos a un proceso laboral psicológicamente intenso, debido a: convivencia con el sufrimiento y la muerte, alta demanda de pacientes, trabajo en turnos, conflictos en las relaciones interpersonales y con el bajo reconocimiento. (p.345)

Es conocido que la presión que experimentan estos trabajadores ya sea por el ritmo de trabajo, las largas jornadas y su relación cercana con los pacientes y sus familias aumentan su nivel de estrés. (Vásquez Trespalacios, Aranda Beltrán y López Palomar,2020, p.333). Por otro lado, la eficacia y los resultados en materia del servicio de salud se evalúan por la calidad de la asistencia que se brinda y entrega a los usuarios, siendo esencial enfocar aquellos elementos que la afectan, como es el caso del Síndrome de Burnout(SB), un fenómeno que perturba directamente la eficacia de la organización sanitaria, produciendo 
Ángela Aracely Andrade-Andrade; Tamara Vidal-Intriago; Grey Marisol Benavidez-Zambrano; Esther Magdalena Gutiérrez-Pibaque

ausentismo laboral, justificativos médicos y disminuyendo la productividad. Este síndrome, además contribuye al deterioro de la calidad de vida laboral en el colectivo, situación que inevitablemente conlleva represiones negativas sobre el servicio de salud. Al respecto, Ramírez (2017) plantea:

Actualmente el concepto de burnout se utiliza para hacer referencia a un tipo de estrés laboral crónico, generado específicamente en profesionales que mantienen una relación y contacto directo con personas, especialmente en relaciones de ayuda. Por ello, uno de los colectivos más afectado es el formado por el personal sanitario (médicos/ as, enfermeros/as, psicólogos/as, psiquiatras, etc.). Este grupo es especialmente vulnerable por la complejidad de las situaciones que deben afrontar en su desempeño diario. (p.242)

En tal sentido, es necesario la gestión del talento humano en las organizaciones hospitalarias para garantizar un desempeño del personal de salud acorde a los requerimientos y necesidades de la institución considerando además los elementos y condiciones mínimas de los médicos y enfermaras(os) que hacen vida en el sistema de salud tanto público como privado, con el propósito de aprovechar al máximo el potencial del factor humano, se ha convertido en una necesidad en esta época y contrarrestar los síntomas del Síndrome de Burnout, organizando las políticas de administración de personal se disminuye lo planteado por Intriago Miranda (2019):

Es además un proceso multicausal y muy complejo, donde se proponen algunas causas, entre las que más figuran: sobrecarga de trabajo, falta de estimulación, aburrimiento, stress, pobre orientación profesional, aislamiento, crisis en el desarrollo de la carrera profesional, limitadas condiciones económicas, horarios nocturnos de rotaciones y guardias, falta de recursos humanos, entre otros. (p.97)

De acuerdo a lo plateado se presenta como objetivo general de la investigación analizar el Síndrome de Burnout presente en la profesión de enfermería en las instituciones de salud del Ecuador. 


\section{METODOLOGÍA}

Desde el paradigma cuantitativo, la presente investigación está enmarcada en un diseño documental, ya que se analiza y estudia una serie de datos obtenidos relacionados al Síndrome de Burnout en el personal de enfermería, la investigación documental es un proceso fundamentado en la indagación, recuperación, examen, crítica e interpretación de datos secundarios, (Arias, 2012); lo que quiere decir los alcanzados y registrados por otros investigadores, en fuente impresas, electrónicas y audiovisuales, entre otras fuentes bibliográficas que apoyan esta investigación documental. La población está constituida por tesis, revistas, trabajos arbitrados; fundamentando el contenido teórico. En relación a la técnica de análisis de contenido, permitió estudiar y considerar las variables, características y dimensiones, generando la relación de las mismas, lo que permitió comparar y contrastar con la realidad y cumplir con el objetivo de la investigación.

\section{RESULTADOS}

De acuerdo a la revisión documental-bibliográfica se presenta a continuación un cuadro resumen que contiene la definición del fenómeno estudiado según la postura de varios autores lo que contribuye a la construcción desde una perspectiva teórica del tema investigado.

\section{Cuadro 1.}

Perspectiva de autores.

\begin{tabular}{|l|l|}
\hline \multicolumn{1}{|c|}{ Autor(es) } & \multicolumn{1}{c|}{ Definición } \\
\hline Torres Toala et al. (2021) & $\begin{array}{l}\text { El Síndrome de Burnout se presenta con mayor } \\
\text { frecuencia en profesionales de contacto directo con } \\
\text { personas como el personal de educación, salud, } \\
\text { cuidadores, atención al público y asuntos sociales. } \\
\text { Afecta generalmente a personas perfeccionistas o } \\
\text { extremadamente responsables y comprometidas. } \\
\text { Los profesionales sanitarios son un colectivo que se } \\
\text { puede encontrar especialmente afectado por este } \\
\text { síndrome debido a su carga laboral, tensiones de } \\
\text { tipo emocional, directamente relacionados con el } \\
\text { ejercicio de su actividad. (p.127) }\end{array}$ \\
\hline
\end{tabular}




\begin{tabular}{|l|l|}
\hline Anzules Guerra, Véliz Zevallos, Vinces Centeno y & $\begin{array}{l}\text { Este síndrome puede ser caracterizado como una } \\
\text { reacción asociada a las condiciones de trabajo } \\
\text { inicialmente identificadas en personas que } \\
\text { desempeñan funciones con intensa interacción } \\
\text { humana, principalmente ligada al área de la salud. } \\
\text { (p.6) }\end{array}$ \\
\hline Pines y Aronsos(1996) & $\begin{array}{l}\text { Señalaron que es el estado de agotamiento } \\
\text { emocional, físico y mental generado por el } \\
\text { involucramiento crónico en el trabajo en situaciones } \\
\text { emocionalmente demandantes.(p.79) }\end{array}$ \\
\hline
\end{tabular}

Elaboración: Los autores.

Así mismo se muestra a continuación los resultados de un trabajo de investigación titulado: Síndrome de Burnout en el personal de enfermería y auxiliares de NovaClínica Santa Cecilia de la ciudad de Quito, Pichincha, Ecuador, durante los meses de octubre 2018-marzo 2019, desarrollado por Fátima Cristina Ágreda Vásquez (2019). En este sentido, el trabajo de investigación del área de Psicología Clínica, cuyo objetivo principal fue determinar el nivel de Síndrome de Burnout que presentaron el personal de enfermería y auxiliares de NovaClínica Santa Cecilia. Para llevar a cabo la investigación, se utilizó una metodología de enfoque cuantitativo, alcance de tipo descriptivo, no experimental y de corte transversal. Se aplicó una encuesta sociodemográfica con datos personales y laborales y, el instrumento Maslach Burnout Inventory (MBI-HSS). Los participantes fueron 84 en total, en donde el $48 \%$ son licenciadas en enfermería y el 52\% son auxiliares. A nivel general se presentó Síndrome de Burnout en el $10,7 \%$ de los participantes. Se encontró que el $47 \%$ presentaron niveles bajos de realización personal, siendo esta la dimensión de riesgo más relevante dentro del estudio. Se definió como perfil de riesgo: mujer, soltera o divorciada, con 2 o más hijos, auxiliar de enfermería, con un rango de edad de 20 a 29 años o, de 50 a 60 años, con un tiempo de servicio de 3 a 5 años y con un empleo. Se recomienda contar con servicios psicológicos para los trabajadores y realizar evaluaciones periódicas para prevenir y tratar la presencia del síndrome. Al respecto se puede detallar los siguiente: 
- La dimensión de Síndrome de Burnout que predomina en el personal de enfermería y auxiliares es la de baja realización personal con un resultado de $47 \%$, mientras que las dimensiones de agotamiento emocional y despersonalización obtuvieron 20 y $33 \%$ respectivamente.

- Las características sociodemográficas y laborales del personal de enfermería y auxiliares, halladas en esta investigación son las siguientes: profesionales entre 2029 años y personas entre 50- 60 años; estado civil divorciado o soltero; dos o más hijos; auxiliar de enfermería; tiempo de servicio en la institución de 3-5 años; un empleo.

- Según las características sociodemográficas y laborales encontradas, se pudo diferenciar quienes son más vulnerables a padecer Síndrome de Burnout y se encontró un perfil de riesgo importante: mujer, soltera o divorciada, con 2, 3 o más hijos, ser auxiliar de enfermería, con un rango de edad de 20 a 29 años o, a su vez, de 50 a 60 años, con un tiempo de servicio de 3 a 5 años y un empleo.

- El nivel de Síndrome de Burnout en el personal de enfermería y auxiliares de NovaClínica Santa Cecilia fue del 10,7\%.

- Se encontró que la mayoría del personal $(80,9 \%)$ tiene bajo o ningún conocimiento acerca del Síndrome de Burnout.

\section{CONCLUSIÓNES}

Al finalizar la investigación se presentan las siguientes alternativas con el objeto de disminuir la presencia y riesgo del Síndrome de Burnout en los recintos de salud. Las instituciones sanitarias deberían de adoptar las siguientes estructuras:

- Horizontal

- Descentralizada

- Con mayor grado de independencia

- Promociones interna justas 
Ángela Aracely Andrade-Andrade; Tamara Vidal-Intriago; Grey Marisol Benavidez-Zambrano;

Esther Magdalena Gutiérrez-Pibaque

- Flexibilidad horaria

- Apoyo a la formación

Algunas acciones preventivas pueden ser:

Reforzar actividades recreativas. Es necesario tener un tiempo para realizar actividades que generen placer y permitan despejar la mente.

Reforzar relaciones personales. Los vínculos sociales funcionan como un apoyo frente a estas situaciones, además de fomentar reforzamiento positivo.

Respetar tiempos. Es fundamental que los tiempos de las comidas y de descanso, sean cumplidos. El tiempo para despejarse del ámbito laboral, permite que la mente rinda más. Diferenciación. Es importante poder diferenciar lo laboral de lo personal. Cuando termina el horario laboral procurar que los problemas relacionados con el trabajo queden allí. No trasladar el trabajo a casa.

Se concluye que es necesario una reorientación de las actividades laborales para lograr un equilibrio emocional, evitar el estrés, y la sobre carga de trabajo evitando la aparición de los síntomas del Burnout.

\section{REFERENCIAS CONSULTADAS}

Arias, F. (2012). El Proyecto de Investigación. Introducción a la metodología científica. Caracas. Venezuela. 5a Edición. Editorial Episteme.

Arias Gallegos, W., y Muñoz del Carpio Toia, A. (2016). Síndrome de burnout en personal de enfermería de Arequipa. Revista Cubana de Salud Pública, 42(4) Recuperado de: https://n9.cl/1x6tm

Ágreda Vásquez, F. (2019) Síndrome de Burnout en el personal de enfermería y auxiliares de NovaClínica Santa Cecilia de la ciudad de Quito, Pichincha, Ecuador, durante los meses de octubre 2018 - marzo 2019. Tesis de pregrado de Psicóloga Clínica. Universidad Central del Ecuador. Ecuador 
Anzules Guerra, J.; Véliz Zevallos, I.; Vinces Centeno, M., y Menéndez Pin, T. (2019). Síndrome de burnout: Un riesgo psicosocial en la atención médica. Revista Arbitrada Interdisciplinaria de Ciencias de la Salud. SALUD Y VIDA, 3(6),1-21. http://dx.doi.org/10.35381/s.v.v3i6.393

Bosqued, M. (2008). Quemados. El síndrome de Burnout. Barcelona. Editorial Paidos.

De Arco Canoles, O. y Suarez Calle, Z. (2018) Rol de los profesionales de enfermería en el sistema de salud colombiano. Univ. Salud, 20(2):171-182. DOI: http://dx.doi.org/10.22267/rus.182002.121

Intriago Miranda, S. (2019). Síndrome del "Burnout" en personal de salud de un centro hospitalario de la dirección distrital Junín-Bolívar. Revista San Gregorio, (35), 94107. https://doi.org/10.36097/rsan.v1i35.962

Pines, A. y Aronsos P. (1996) Burnout en profesores: Impacto sobre la calidad del a educación, la salud y consecuencias para el desarrollo profesional. Memoria final de investigación. Madrid. MEC-CIDE

Ramírez, M. (2017). Prevalencia del síndrome de burnout y la asociación con variables sociodemográficas y laborales en una provincia de ecuador. International Journal of Developmental and Educational Psychology, 4(1),241-251. Recuperado de: https://n9.cl/ei6h5

Rodríguez, D. (2005) Análisis del clima organizacional en la secretaria de comunicaciones transporte centro Colima. Tesis de maestría en administración. Universidad de Colima. Argentina.

Torres Toala, F., Irigoyen Piñeiros, V., Moreno, A., Ruilova Coronel, E., Casares Tamayo, J., y Mendoza Mallea, M. (2021). Síndrome de Burnout en profesionales de la salud del Ecuador y factores asociados en tiempos de pandemia. Revista Virtual de la Sociedad Paraguaya de Medicina Interna, 8(1), 126 136..https://dx.doi.org/10.18004/rvspmi/2312-3893/2021.08.01.126

Vásquez Trespalacios, E., Aranda Beltrán, C., y López Palomar, M. (2020). Síndrome de Burnout y justicia organizacional en profesionales de enfermería de hospitales de tercer nivel en la ciudad de Medellín. Revista de la Asociación Española de Especialistas en Medicina del Trabajo, 29(4), 330-339. Recuperado de: https://n9.cl/9z2p0 


\section{Revista Interdisciplinaria de Humanidades, Educación, Ciencia y Tecnología}

Año VII. Vol. VII. N². Edición Especial II. 2021

Hecho el depósito de ley: pp201602FA4721

ISSN-L: 2542-3029; ISSN: 2610-802X

Universidad Nacional Experimental Francisco de Miranda (UNEFM). Santa Ana de Coro. Venezuela

Ángela Aracely Andrade-Andrade; Tamara Vidal-Intriago; Grey Marisol Benavidez-Zambrano;

Esther Magdalena Gutiérrez-Pibaque

Vidotti, V., Martins, J., Galdino, M., Ribeiro, P., y Robazzi, M. (2019). Síndrome de burnout, estrés laboral y calidad de vida en trabajadores de enfermería. Enfermería Global, 18(3), 344-376. https://doi.org/10.6018/eglobal.18.3.325961

(C2021 por los autores. Este artículo es de acceso abierto y distribuido según los términos y condiciones de la licencia Creative Commons Atribución-NoComercial-Compartirlgual 4.0 Internacional (CC BY-NC-SA 4.0) (https://creativecommons.org/licenses/by-nc-sa/4.0/ 